\title{
Integrating emotional affect into bear viewing management and bear safety education
}

$\underline{\text { John M. Nettles }}^{1}$, Matthew T. J. Brownlee $^{1}$, Jeffrey C. Hallo ${ }^{1}$, David S. Jachowski ${ }^{2}$ and Ryan L. Sharp ${ }^{3}$

\begin{abstract}
The popularity of viewing wildlife, specifically brown bears (Ursus arctos), is increasing rapidly throughout North America. In addition, population distributions of both humans and brown bears are expanding, creating larger areas of overlap and an increased possibility of human-bear interactions. In order to prevent negative encounters and injury to either species, park managers must continue to work to encourage appropriate behavior among local citizens as well as park visitors. Human behavior, however, is a result of many complex factors, including emotion and cognition. Despite this, the effects of emotions on human-wildlife conflict remain unstudied and therefore may limit success of any mitigation efforts. In this study we employed a quantitative self-assessment questionnaire, distributed online to a representative sample of the general U.S. public, to understand the relationship between emotion and behavior within the context of human encounters with bears. Questionnaires used video clips as visual methods to illustrate a variety of brown bear encounter scenarios based on setting, the bear's age or sex class, and bear behavior. Following each video, respondents were asked to rate the intensity of their affective responses using the Positive and Negative Affect Schedule and then rate the likelihood of performing several listed actions as well as the perceived appropriateness of each action. Results demonstrate significant variation in negative affect and relative consistency in positive affect across brown bear encounter scenarios. In general, respondents seemed to be aware of appropriate behavior during encounters with brown bears, but affective responses may limit their ability to behave accordingly. Further, feelings of fear and hostility increased the impact of current emotion on in-the-moment decisions. These results and suggestions provided by respondents were then used to create a set of meaningful recommendations to improve the efficacy of current bear management and safety education.
\end{abstract}

Key Words: affect; bear safety; brown bears; emotion; human behavior; human-wildlife conflict

\section{INTRODUCTION}

Already one of the most popular forms of ecotourism, bearviewing continues to grow in popularity (Aumiller and Matt 1994, Haroldson and Gunther 2013, Penteriani et al. 2017). The creation of Yellowstone National Park (NP) in 1872 inspired some of the earliest documentation of recreational bear-viewers (Aumiller and Matt 1994), and as interest increases, destinations such as McNeil River State Game Sanctuary in Alaska develop into high demand tourist attractions. This site, specifically, provides a guiding example of successful bear-viewing management. After the establishment of their management plan, the sanctuary touts no human injuries or bears requiring removal (lethal or otherwise), despite bear use of the area more than doubling (Aumiller and Matt 1994, Penteriani et al. 2017). Managers at McNeil River claim this is largely a result of their ability to encourage appropriate behavior among visitors so that human behavior appears predictable to foraging bears.

\section{Previous bear attacks}

Unfortunately, when park visitors and local citizens behave inappropriately, both bears and humans can be harmed. Although injurious encounters with bears are relatively rare, the result can prove fatal. Canadian biologist, Stephen Herrero (2018) found that from 1900 to 1980,126 injuries were definitely or very probably inflicted by grizzly bears in North American parks. Of these, 56 occurred in Yellowstone National Park and 24 occurred in Glacier National Park. However, two parks with much larger bear populations, Katmai and Denali National Parks \& Preserves in Alaska, accounted for only 10 injuries during this time period, all of which were in Denali (Herrero 2018). Between 1970 and 1973, Yellowstone reported the highest ratio of visitors per grizzly-inflicted injury at $1,745,142: 1$, or approximately $0.00006 \%$ of the visiting population (Herrero 1976).

In this same time period, sows with cubs were responsible for eight major injuries and nine minor injuries, while solitary females were responsible for one major injury and adult males were responsible for one major and one minor injury (Herrero 1976). Among 50 aggressive encounters not resulting in injury, 28 involved a sow with cubs, two involved an adult male (five additional accounts attributed to an adult bear of unknown sex), and five involved a subadult (Herrero 1976). Overall, these data suggest interior parks with high visitation and relatively small bear populations produce the highest risk of bear-inflicted injury which, if occurred, would most likely be attributed to a sow with cubs. Despite this potential for danger, visitors often hope for the special experience of being near animals or seeing dependent young (Farber and Hall 2007, Verbos et al. 2018, Nettles et al. 2020).

Several park visitor elements can influence the likelihood of human-wildlife conflict, including visitor density, visitor distribution, visitor activity type, resource consumption, and visitor transportation (Monz and Leung 2006). Related to human-bear conflicts, visitor distance to a bear ( $<50$ meters), appropriateness of visitor behavior, and predictability of visitor behavior have all proved important factors for the occurrence and frequency of bear attacks (Hammitt et al. 2015). For example, in wildlife viewing scenarios where park visitors perform the same

${ }^{1}$ Park Solutions Lab at the Clemson University Institute for Parks, Department of Parks, Recreation, and Tourism Management, Clemson University, Clemson, SC, USA, ${ }^{2}$ Department of Forestry and Environmental Conservation, Clemson University, Clemson, SC, USA, ${ }^{3}$ Applied Park Science Lab, Department of Horticulture and Natural Resources, Kansas State University, Manhattan, KS, USA 
actions during each visit, i.e., predictability, regardless of the diversity of visitors, researchers and managers can expect to reduce the level of human-wildlife conflict to be avoided (Hammitt et al. 2015).

\section{Emotion and behavior}

During encounters with wildlife, humans' emotions are key determinants of their decision making yet are seldom studied. A large majority of these encounters are novel or unexpected, potentially increasing the severity of this relationship. Excitement, anxiety, anger, or simply the desire to capture a highquality photograph, may override any previous knowledge of appropriate behavior. Despite this, little knowledge exists regarding the impact of humans' emotions on the management of human-wildlife conflict. As a result, management strategies might be less effective, potentially limiting the success of current preventative education efforts. Thus, human-wildlife conflict management can improve only if human emotions are assessed in developing future education strategies that target both their subconscious and conscious reactions to wildlife. We sought to explore human reactions to specific wildlife encounter scenarios and their potential implications, focusing on responses to bearviewing among the general American public. Many visitors and residents of bear-inhabited regions never receive formal bear safety education and as populations of both bears and humans expand, negative encounters may continue. Collecting a representative sample of the general American public allows for an unbiased estimate of national awareness regarding safe behavior around bears and potential suggestions for improvement.

The lack of empirical studies investigating the role of human emotion in human-bear conflicts, and the need to improve human-wildlife conflict management strategies underpinned this study. Furthermore, the influence of setting, and bear age and sex, on human emotions and subsequent decision making has not been addressed to date. Therefore, the objectives of this paper include to (1) understand potential emotional reactions upon viewing bears in different treatment scenarios, including various sexes, age classes, behaviors, and habitat types, and (2) understand the role of emotional reactions in individuals' ability to behave appropriately. Research questions include the following:

1. How do affective responses to the setting, bear behavior, and a bear's sex or age class impact decision-making among bearviewers? 2. How do affective responses vary across treatments?

3. How does the likelihood of performing listed actions vary across treatments?

4. How does the reported appropriateness of performing listed actions vary across treatments?

5. For which behaviors are there discrepancies between likelihood and reported appropriateness?1. What factors may contribute to the decision-making process during a bear encounter?

\section{BACKGROUND}

Underpinning these research questions is a body of knowledge about emotion, mood, and affect, which despite similar connotations, all represent different phenomena. As described by
Ekkekakis (2012), affect, the simplest of the three, represents a primitive reaction or response to a stimulus and can occur alone or as a component of mood or emotion. Emotion is the complex interaction between sub-events regarding a specific object. These sub-events include core affect, overt behavior, directed attention, cognitive appraisal of a stimulus, connection of emotion to a stimulus, experience of the emotion, and neural and endocrine changes. Unlike affect and mood, emotion requires cognitive awareness and thought. Last, mood is similar to emotion but often persists much longer and is more abstract, lacking a specific or immediate cause (Ekkekakis 2012).

Two judgmental heuristics - practical approaches to decision making, often separated from logic or rationale - may help understand human behavior through understanding mental predictions. The first, representativeness, involves an individual predicting the outcome best represented by the evidence. These intuitive predictions often ignore reliability of the evidence and lead individuals to predict rare events if they happen to be representative (Kahneman and Tversky 1973). Second is availability, or the process of decision making by the ease at which relevant solutions come to mind. This leads to systematic biases represented through frequencies of word classes, combinatorial outcomes, and repeated events (Tversky and Kahneman 1973). The use of these heuristics can be detrimental when presented with previously unknown situations, such as instances of humanwildlife encounters. The decision, and resulting action, may not be the logical or reasonable solution, but simply the most representative or first available (Kahneman and Tversky 1973 , Tversky and Kahneman 1973).

Traditional research in judgment and decision making focused on cognitive processes as the basis for uncertain decisions, in both microeconomics and philosophy (Quartz 2009). However, starting in the 1990s, research began to incorporate emotional processes within decision making. Rather than the previous dichotomy between emotional and cognitive function, the two may be indistinguishable. Emotions not only encode heuristic evaluations, but the precise parameters of cognitive ones as well (Slovic et al. 2005). These evaluations are often referred to as the experiential and analytic systems, respectively. The experiential system provides fast, nearly automatic decisions, while the analytic system provides slow, effortful, and conscious decisions. Previously the experiential system received less credit than the analytic system but current wisdom states both are required in rational decision making. Therefore, when encouraging rational decisions, both systems must be addressed (Quartz 2009).

In order to reach rational, successful decisions, both cognitive and emotional aspects must be addressed. Emotion not only informs heuristic decision-making processes, but cognitive processes as well, and effective conflict management requires implementation of both to better inform future actions and reasoned decision making. Integrating such complexity in management efforts requires a multi-disciplinary approach. Items impacting decision making during an encounter include cognition and emotions, barriers and benefits to specific behavior choices, and social thresholds (Jochum et al. 2014). These dimensions each play a role in determining behavior; however, some may lack effectiveness because of individuals' reliance on affect-based shortcuts. 
Wilson (2008) used three experiments to test individuals' ability to overcome affect-based shortcuts and balance cognitive and emotional processes when making decisions. The first demonstrated participants' allocation of hypothetical conservation funds according to affective impact rather than accordance with their personal rankings of management objectives. Value-focused approaches aim to address this by incorporating an individual's conservation objectives into their decision-making process. The second experiment demonstrated individuals' reliance on affect, even when it contradicted statistical evidence. The affective response of fear encouraged individuals to choose a management solution with a proven greater physical risk. Trade-off techniques address this by encouraging justification for conservation actions over affective impressions or other values. Last, the third experiment demonstrated that individuals felt they could accurately estimate the losses of strangers, but strangers could not accurately estimate theirs. This distrust in decision-making authorities can significantly hamper the success of a decision. Identifying shared values and increasing procedural fairness work to address this by fostering trust in decision-making authorities and the decision process (Wilson 2008). Through incorporation of these techniques, managers develop the capacity to edit individuals' affective responses to wildlife encounters, quickly and drastically altering the effectiveness of mitigation efforts. Despite this, current bear safety instruction as outlined by Herrero (2018) and Masterson (2016), lacks consistent application of such tactics. As Zajchowski et al. (2016) recommend, incorporating the knowledge of heuristic processing into the management of outdoor recreation participants and activities is likely a key determinant of safe and successful outdoor pursuits.

\section{METHODS}

\section{Overall design}

To best determine how the American public responds emotionally to various bear-encounter scenarios, we used an exploratory sequential design (Cabrera 2011). The first phase of our research involved informal in-situ interviews with bear-viewers at Katmai and Lake Clark National Parks and Preserves in Alaska. During this same period, we recorded different bear age, sex, and behavior using field video cameras. The second phase involved developing an online survey instrument to quantitatively capture affective responses to various bear encounter scenarios using two quasiexperimental designs. In the third phase, we administered the survey instrument to a representative sample of the general American public using a cross-sectional representative sample. The fourth and final phase consisted of the statistical analysis of survey results to address the research question.

This study employed two independent samples designs: a $3 \times 3$ design as well as an additional $3 \times 1$ design. Each treatment represented a different 15 -second video. The first design $(3 \times 3)$, setting treatments, compared visitor responses across three different settings and three different age and sex classes. Settings included a bear either in a salmon stream, a meadow, or a viewing platform while age and sex classes included solitary boar, sow with cubs, and sub-adults. Study design 2 ( $3 \times 1)$, behavior treatments, acted as a qualifier, testing responses across bear behavior, holding other variables constant. The three studied behaviors were feeding, curious, and aggressive, but respondents were not provided with an interpretation or description of these behaviors.
Behavior was held constant across the entire $3 \times 3$ design but was tested here to alleviate the threat to external validity resulting from the lack of independence between bear behavior and an individual's response. This second design also served as an additional test of current education efforts and an individual's ability to behave appropriately in such situations.

\section{Phase 1: Informal interviews}

\section{Informal interviews}

Brief qualitative interviews occurred directly after or during a bear-viewing experience, following approaches recommended by Seidman (2012). These interviews were informal, recorded only through field notes, and looked to capture the current emotions of visitors (Halcomb and Davidson 2006). Questions were open ended to allow the respondent to verbalize their reactions independent of outside influence ${ }^{[1]}$. Interviews were conducted with approximately 45 visitor groups across multiple locations within Katmai and Lake Clark National Parks to reach data saturation (Seidman 2012). These locations support abundant brown bear (Ursus arctos) populations while providing high quality viewing experiences in all three of the intended settings (Nettles et al. 2020).

\section{Video capture}

To best simulate the nine setting treatments, we captured high quality video clips that effectively demonstrated the intended scenario while minimizing any confounding variables due to unintended differences across videos, such as lighting, weather, microhabitat, size and positioning of the bear, and video quality. For the three behavior treatments, we elicited videos from online bear-viewing communities, online video sharing sites, and public access documentaries. Videos were selected that displayed only the focal bear and clearly illustrated the intended behavior while minimizing differences due to microhabitat, lighting, weather, age or sex of the bear, and video quality. All final videos are available online (https://doi.org/10.6084/m9.figshare.c.5296201.v1).

Researchers in park and visitor use management often use visual methods, in the form of computer-altered photographs, to help identify outdoor recreationists' ideal conditions (Laven and Krymkowski 2005, Cribbs et al. 2019). Photographs depicting varying levels of resource or experiential impact can be used as suggestive surrogates for actual conditions (Newman et al. 2001) and are much more effective than traditional narrative descriptions (Manning and Freimund 2004). Although less studied, videos have also been used successfully to determine park visitors' preferred conditions (Bateson and Hui 1992, Freimund et al. 2002). This study would have ideally been conducted in person, but field research would not have allowed the questions to be addressed. Not only would this method be time consuming and expensive, it would be difficult to maintain consistency across scenarios and ensure all types of encounters were represented. As a result, videos were used to best depict first-hand experience of bear behavior and viewing locations, allowing us to design the experiment appropriately while keeping both bears and humans safe and avoiding distractions during onsite experiences.

\section{Phase 2: Survey development}

For the second phase of the design, we developed an online selfassessment survey of emotional affect toward brown bears among the general American public. Surveys used the Positive and 
Negative Affect Schedule (PANAS scale) to quantify affective reactions of survey respondents. Developed by Watson et al. (1988), the PANAS scale is one of the most widely used tools for measuring affect (e.g., Crocker 1997, Schmukle et al. 2002, Crawford and Henry 2004, Thompson 2007, Jacobs et al. 2012a). This scale uses a list of 20 adjectives describing various emotions, 10 of which relate to positive affect, and 10 to negative affect. Respondents are asked to rate the intensity of each affective item on a five-point scale, with one being "very slightly or not at all" and five being "extremely." This scale has consistently exhibited high scale, item, and external validity, is reliable across a range of time instructions from the present moment to general, and provides precise measures of positive and negative affect (Watson et al. 1988).

We included photographs of viewing scenarios and explicit instructions in the survey to encourage respondents to immerse themselves in the video and respond based only on immediate reactions to each video. Figure 1 displays the photographs for each setting category, i.e., meadow, stream, and platform. This figure also includes the generic bear viewing photo displayed prior to all three behavior treatments used to further illustrate the viewing scenario.

Fig. 1. Images shown to respondents prior to viewing the respective setting and behavior treatment videos.

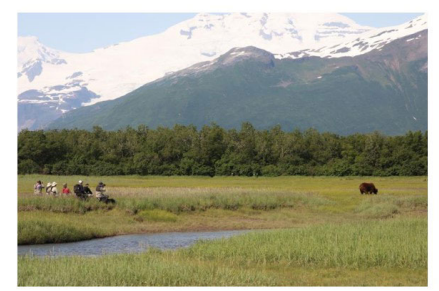

Meadow

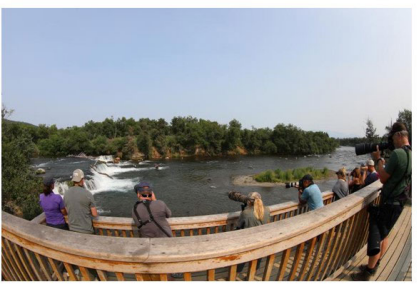

Platform

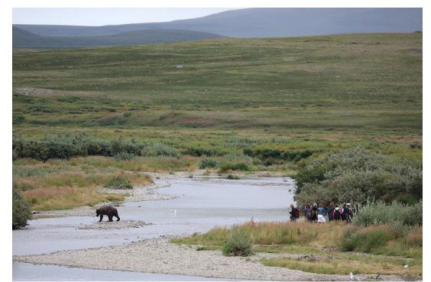

Stream

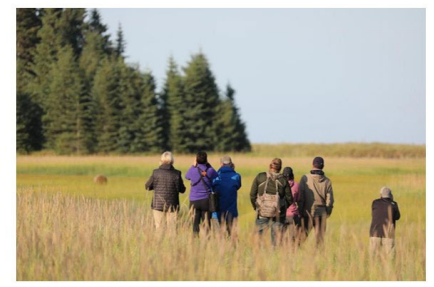

Behavior
We developed the survey ${ }^{[2]}$ through an online survey platform, Qualtrics, using best practices for online survey construction as described by Dillman (2011) and Vaske (2008). Surveys allowed each respondent to view a randomly selected video from the first design, a $3 \times 3$ design with three settings and three categories of sex or age class, followed by a question prompting them to rate their level of agreement with all listed adjectives, using the PANAS scale to measure positive and negative affect. Next, surveys showed each respondent a randomly selected video from the second design, a $3 \times 1$ design with three bear behavioral categories, followed by the same PANAS scale. Despite efforts to maximize the applicability of videos and the PANAS scale, their use may have hampered the ability to measure true affective responses. This study, then, offers a foundation for future research that could use in-depth, onsite, qualitative interviews to better understand the complexities within affective responses.

For each video, respondents were asked how they plan to react and the perceived appropriateness of potential actions. Both questions provided a list of 15 potential actions and a commonly used seven-point balanced Likert scale for respondents to rate their level of likeliness and appropriateness of each action (ranging from 1 = extremely unlikely or extremely inappropriate to $7=$ extremely likely or extremely appropriate). These potential actions were chosen based on historical accounts of reactions listed by Gunther and Hoekstra (1998) and personal accounts from working in bear safety education. The final list was chosen to provide a range of appropriateness and was listed in alphabetical order. Last, the respondents rated, on a 10-point Likert scale $(1=$ not at all, $10=$ a great deal $)$, the potential impact of bear safety education, current emotion, and previous experience on their in-the-moment decisions. These questions helped to determine the strength and accuracy of judgmental heuristics, i.e., representativeness and availability, in respondents' decision-making process.

\section{Phase 3: Sampling}

After developing the final survey instrument, we used Qualtrics to collect a representative sample of the general American public. Unless bear safety training is mandatory and standardized, many visitors and residents may encounter a bear without having knowledge of proper behavior. We chose the general American public as the study population to best understand how such individuals will behave in bear encounter scenarios, with or without previous training. An online cross-sectional representative sample allowed for efficient data collection given the diverse intended population. Qualtrics sampling used six demographic questions and standard U.S. Census Bureau categories as qualifiers to ensure a representative sample of the American public. These included age, education level, gender, income, race, and zip code of primary residence.

\section{Phase 4: Analysis}

All statistical analyses were conducted using IBM's Statistics Package for the Social Sciences (SPSS) version 24.0 (2018). To confirm that the sample produced enough statistical power to detect differences in means, we calculated the minimum sample size per cell needed to maintain a power of 0.8 . Because statistical power decreases with further segmentation, we determined the minimum sample size required for each of nine cells within design 1 , i.e., setting treatments. If the true effect size was 0.2 , each cell would require a sample size of 43 to produce a power of 0.8 at a significance level of 0.05 . If the true effect size was 0.15 , each cell would require a sample size of 75 .

To determine fit indices, measurement variance, and item independence for the shortened PANAS scale, we conducted a confirmatory factor analysis (CFA) with robust estimation methods using EQS 6.3. Ultimately, the CFA helps evaluate the psychometric measurement properties of the scale and provides an assessment of scale validation.

To assess the difference in intensity of affective responses (question 1a), likelihood of performing listed actions (question 1b), and perceived appropriateness of listed actions (question 1c), we used multivariate analyses of variance (MANOVAs). When 
comparing across behavior treatments, we used an individual's previous response as a covariate to account for scale redundancy or dependency due to the repeated measure. When a difference across video treatments was statistically significant $(p<0.05)$, we used the conservative Bonferroni post-hoc test to assess pair-wise comparisons while minimizing the impact of testing multiple hypotheses on statistical results.

To assess the difference in an individual's likelihood of performing listed actions and perceived appropriateness of listed actions (question 1d), we used paired-samples t-tests. Because each individual was only shown one combination of video treatments and the impact of a previous scale response is likely similar for an individual across questions, the impact of previous scale completion on an individual's responses to these two questions is limited. As a result, we did not include previous responses as a covariate when testing across behavior treatments. In terms of the mean difference, a negative value represents an action that is more likely than appropriate, while a positive value represents an action that is more appropriate than likely.

For all analyses using covariates, we calculated the two-tailed Pearson correlation coefficient, $r$, to confirm the need for their inclusion. This value represents the level of covariation between the variable of interest for the setting and sex or age class treatment and that for the behavior treatment. Values of $r$ between 0.7 and 1.0 or -0.7 and -1.0 represent strong positive or negative linear relationships, respectively.

To determine which factors contribute most to an individual's decision-making, we conducted a univariate general linear model (GLM). We included demographics, experience use history, and affective responses in the global model, removing variables to maximize the adjusted $\mathrm{R}^{2}$ value. For all three final models, we included video treatment, regardless of statistical significance to illustrate its impact, or lack thereof, on respondents' likelihood of decisions.

\section{RESULTS}

We received a total of 511 complete responses with approximately 57 responses $(S D=4.39)$ for each of nine setting treatments and 170 responses $(S D=7.76)$ for each of three behavior treatments. For all sample-wide analyses, this produced an overall confidence interval of $4.34 \%$ at the $95 \%$ confidence level, which along with demographic matches suggests a representative sample of the American public. Based on the average observed sample size per cell $(n=57)$, an effect size of 0.15 , and a significance level of 0.05 , the resulting statistical power was 0.66 . As a result, we were confident in the sample's ability to detect even minor differences in means.

The sample accurately represented the general American public, as estimated by the U.S. Census Bureau (USCB 2020), with $66.5 \%$ identifying as white, $16.6 \%$ as Hispanic or Latino/Latina, $18.4 \%$ earning less than $\$ 24,999$ per year, $17.8 \%$ earning between $\$ 50,000$ and $\$ 74,999,14.9 \%$ earning between $\$ 25,000$ and $\$ 49,999,24.1 \%$ reporting high school graduate as their highest level of education, $24.1 \%$ reporting a four-year college degree as their highest level of education, and $45 \%$ identifying as female. Of these responses, $55 \%$ reported never seeing a wild brown bear, while $11.2 \%$ reported seeing a wild bear but were unsure of the species. In addition, $47.2 \%$ reported being taught any form of bear safety.
However, it is unlikely that $33 \%$ of the sample truly saw a wild brown bear. Although it does not impact the validity of results, it does suggest that the American public may respond to black and brown bears in the same manner, or lack the ability to accurately identify different types of bears.

\section{Informal interviews}

As discussed above, the survey used an adjusted version of the PANAS scale, one of the most widely used scales to measure individual affect. To minimize burden on the respondent and eliminate items not relevant to a bear viewing experience, we included only adjectives described in the qualitative interviews while maintaining balance between the positive and negative sides. This process resulted in use of 10 of the 20 PANAS items. The five chosen items within positive affect were attentive, alert, enthusiastic, excited, and interested. The five chosen items within negative affect were distressed, hostile, jittery, nervous, and scared.

\section{Confirmatory factor analysis}

Results of the original CFA demonstrated low factor loadings and cross loadings for two positive affect items: alert and attentive (initial model fit indices: $\mathrm{SB} \chi^{2}=1158, p<0.01, \mathrm{CFI}=0.82$, NNFI $=0.82$, RMSEA $=0.18$, SRMR $=0.181)$. As a result, we conducted an additional CFA, excluding alert and attentive. This model resulted in relatively high factor loadings, with 6 of the 7 items above 0.76 and one at 0.54 (modified model fit indices: $\mathrm{SB} \chi^{2}$ $=122.7, p<0.01, \mathrm{CFI}=0.98, \mathrm{NNFI}=0.97, \mathrm{RMSEA}=0.07$, SRMR = 0.042). Intuitively, alert and attentive are not limited to positive responses, which might have influenced the cross loadings and low factor loadings. However, alert and attentive were considered by researchers (e.g., Watson et al. 1988) to be positive affect items.

Although this second model met the customary levels of fit as described by Byrne (2008) and Kline (2012), these authors advise researchers to interpret fit indices holistically, maintaining theoretical and conceptual validity. Because results for each item were foundational to this study, we chose to include alert and attentive in all analyses except for those at the factor level of either positive or negative affect. Ultimately, the CFA results suggest that most PANAS items (a) appropriately reflected the intended latent variables or factors of positive affect and negative affect, (b) exhibited appropriate convergent validity, and (c) that comprised the factors allowed the constructs to achieve acceptable divergence or independence.

\section{Research question 1: Responses to setting, bear behavior, and bear sex or age class}

Question 1a: Affective responses: setting, bear sex and age class, and bear behavior

Among the Positive Affect (PA) items, alert and excited varied the most across setting treatments but none were statistically different at the $p>0.05$ level. Among the Negative Affect (NA) items, distressed, jittery, nervous, and scared were all statistically different across setting treatments (Table 1). Overall, viewing a sow and cubs in a meadow resulted in a statistically stronger negative reaction than viewing a boar in a meadow, a boar from a platform, or a subadult from a platform.

Across all positive and negative affect items, intensity differed significantly across behavior treatments (Table 2). For three 
Table 1. Mean intensity of affective response to the nine setting treatments. Note: Intensity was ranked by respondents on a five-point scale with 1 representing "very slightly or not at all" and 5 representing "extremely." Superscripts represent significant differences across rows at the $\mathrm{p}<0.05$ level. Total PA does not include alert or attentive. ${ }^{*} \mathrm{p}<0.05 * * \mathrm{p}<0.01$

\begin{tabular}{|c|c|c|c|c|c|c|c|c|c|c|}
\hline & Boar & $\begin{array}{c}\text { Sow and } \\
\text { Cubs }\end{array}$ & Subadult & Boar & $\begin{array}{c}\text { Sow and } \\
\text { Cubs }\end{array}$ & Subadult & Boar & $\begin{array}{c}\text { Sow and } \\
\text { Cubs }\end{array}$ & Subadult & \multirow[b]{2}{*}{$F(8,502$} \\
\hline & \multicolumn{3}{|c|}{ Meadow } & \multicolumn{3}{|c|}{ Stream } & \multicolumn{3}{|c|}{ Platform } & \\
\hline & Mean (SD) & & & & & & & & & \\
\hline \multicolumn{11}{|c|}{ Positive Affect } \\
\hline Alert & $3.89(1.2)$ & $3.73(1.2)$ & $3.95(1.1)$ & $3.91(1.2)$ & $3.69(1.4)$ & $3.69(1.3)$ & $3.35(1.1)$ & $3.49(1.3)$ & $3.50(1.4)$ & 1.61 \\
\hline Attentive & $3.93(1.1)$ & $3.80(1.1)$ & $4.22(0.9)$ & $3.93(1.2)$ & $3.72(1.3)$ & $3.86(1.3)$ & $3.68(1.1)$ & $3.84(1.2)$ & $3.75(1.3)$ & 1.16 \\
\hline Enthusiastic & $3.04(1.2)$ & $3.31(1.4)$ & $3.42(1.3)$ & $3.16(1.3)$ & $3.24(1.3)$ & $2.98(1.4)$ & $3.40(1.2)$ & $3.41(1.4)$ & $3.40(1.2)$ & 0.94 \\
\hline Excited & $3.37(1.2)$ & $3.53(1.1)$ & $3.78(1.1)$ & $3.25(1.2)$ & $3.36(1.4)$ & $3.30(1.4)$ & $3.75(1.2)$ & $3.52(1.3)$ & $3.79(1.1)$ & 1.67 \\
\hline Interested & $3.80(1.2)$ & $3.95(1.1)$ & $3.92(1.2)$ & $3.82(1.0)$ & $3.74(1.4)$ & $3.54(1.4)$ & $3.88(1.1)$ & $3.89(1.2)$ & $3.90(1.4)$ & 0.60 \\
\hline Total PA & $10.20(3.0)$ & $10.78(3.2)$ & $11.13(3.1)$ & $10.24(3.3)$ & $10.34(3.6)$ & $9.82(3.8)$ & $11.03(2.9)$ & $10.82(3.4)$ & $11.08(3.2)$ & 1.13 \\
\hline \multicolumn{11}{|c|}{ Negative Affect } \\
\hline Distressed & $1.8(1.0)$ & $2.42(1.3)^{\mathrm{b}}$ & $2.02(1.1)$ & $\begin{array}{l}2.15 \\
(14)\end{array}$ & $1.97(1.1)$ & $2.29(1.4)$ & $1.70(1.1)$ & $1.67(1.2)^{\mathrm{a}}$ & $2.02(1.1)$ & $2.53 * *$ \\
\hline Hostile & $1.69(1.8)$ & $2.13(1.4)$ & $1.73(1.2)$ & $1.64(1.1)$ & $1.52(1.0)$ & $1.88(1.3)$ & $1.55(1.0)$ & $1.62(1.2)$ & $1.98(1.3)$ & 1.65 \\
\hline Jittery & $1.94(1.1)^{\mathrm{a}}$ & $2.76(1.2)^{b}$ & $2.42(1.2)$ & $2.25(1.4)$ & $2.22(1.3)$ & $2.43(1.3)$ & $1.97(1.2)^{\mathrm{a}}$ & $1.80(1.4)^{\mathrm{a}}$ & $2.15(1.3)$ & $3.10^{* *}$ \\
\hline Nervous & $2.22(1.1)^{\mathrm{a}}$ & $3.13(1.3)^{\mathrm{b}}$ & $2.61(1.3)$ & $2.49(1.4)$ & $2.43(1.3)$ & $2.43(1.4)$ & $1.87(1.1)^{\mathrm{a}}$ & $1.92(1.3)^{\mathrm{a}}$ & $2.35(1.4)$ & $4.93 * *$ \\
\hline Scared & $2.22(1.2)$ & $2.96(1.4)^{\mathrm{b}}$ & $2.72(1.2)$ & $2.44(1.4)$ & $2.16(1.1)^{\mathrm{a}}$ & $2.64(1.4)$ & $2.00(1.2)^{\mathrm{a}}$ & $1.92(1.3)^{\mathrm{a}}$ & $2.56(1.5)$ & $4.34 * *$ \\
\hline Total NA & $9.87(4.3)^{\mathrm{a}}$ & $13.40(5.4)^{\mathrm{b}}$ & $11.50(5.1)$ & $10.96(5.9)$ & $10.29(4.8)$ & $11.66(6.3)$ & $9.08(5.7)^{\mathrm{a}}$ & $8.93(5.7)^{\mathrm{a}}$ & $11.06(5.8)$ & $3.91 * *$ \\
\hline
\end{tabular}

positive items, enthusiastic, excited, and interested, the intensity of affective responses decreased statistically with more aggressive behaviors. However, for the other two positive items, alert, and attentive, affective responses to the feeding video were statistically less intense compared to both the curious and aggressive videos. For all items within negative affect, intensity increased statistically $(p<0.001)$ with more aggressive behaviors $(\mathrm{F}[2,507]=$ Distressed: 71.72; Hostile: 26.48; Jittery: 55.58; Nervous: 41.41; Scared: 43.48)

\section{Questions $1 b$ and 1c: Likelihood and appropriateness of} performing listed actions

When asked to rate the likelihood of performing each of several potential actions on a scale from one to seven $(1=$ extremely unlikely, 7 = extremely likely), the most likely actions were back away slowly $(M=5.37, S D=1.8)$, group together $(M=4.16, S D$ $=2.0)$, stand still and wait for the bear to leave $(M=4.73, S D=$ $1.8)$, and walk around the bear $(M=4.51, S D=2.2)$. However, the actions perceived as most appropriate, on a scale of one to seven $(1=$ extremely inappropriate, $7=$ extremely appropriate $)$, were back away slowly $(M=5.57, S D=1.6)$, group together $(M$ $=4.33, S D=2.0)$, stand still and wait for the bear to leave $(M=$ $4.77, S D=1.8)$, try to hide $(M=4.09, S D=2.0)$, and walk around the bear $(M=4.64, S D=2.1)$. For likelihood of listed actions, 12 of 15 actions were significantly different across bear behavior treatments (climb a tree, kick or punch the bear, group together, play dead, run away, shout or make noise, shoot the bear, spray with bear spray, throw food into the woods, throw things at the bear, and try to hide). However, no actions were different across setting and bear age and sex class. ${ }^{[3]}$

\section{Question 1d: Discrepancies between likelihood and perceived appropriateness}

Of the 135 combinations of setting videos and potential actions, 15 showed statistical differences between likelihood and perceived appropriateness, meaning respondents may be aware of correct behavior but are either unable to resist participating in inappropriate behavior (i.e., more likely than appropriate; negative value) or unwilling to participate in appropriate behavior (i.e., more appropriate than likely; positive value). Across all setting treatments, backing away slowly, $t(498)=2.95, p=0.003$, climbing a tree, $t(481)=3.82, p<0.001$, grouping together to appear larger, $t(482)=2.40, p=0.017$, playing dead, $t(486)=4.49$, $p<0.001$, and walking around the bear, $t(476)=2.41, p=0.017$, were generally seen as more appropriate than likely. ${ }^{[3]}$

Of the 45 combinations of behavior video and potential actions provided, seven showed statistical differences between likelihood and perceived appropriateness. Across all behavior treatments, climbing a tree, $t(484)=3.01, p=0.003$, grouping together to appear larger, $t(477)=2.47 p=0.014$, playing dead, $t(482)=3.86$, $p<0.001$, trying to hide, $t(481)=2.59, p=0.010$, and walking around the bear, $t(477)=3.77, p<0.001$, were generally seen as more appropriate than likely. ${ }^{[3]}$

\section{Research question 2: Factors in decision making}

Bear safety education

For the impact of bear safety knowledge on a respondent's inthe-moment decision, seven independent variables were statistically significant, $F(19,1002)=12.75, p<0.001$, adjusted $\mathrm{R}^{2}=0.179$ (see Table 3 ). These were previous bear safety instruction, general feelings toward bears, respondent age, and the affective items of alert, attentive, interested, and hostile. The impact of bear safety education was greater among respondents who had participated in a bear safety training. Additionally, increased positive feelings toward bears, respondent age, alertness, attentiveness, and interest all resulted in an increased impact of bear safety education on decision making. However, increased feelings of hostility decreased the impact of safety education. 
Table 2. Estimated marginal mean for intensity of affective responses to the three behavior treatments. Note: Intensity was ranked by respondents on a five-point scale with one representing "very slightly or not at all" and five representing "extremely." Superscripts represent significant differences across rows at the $p<0.05$ level. The two-tailed Pearson correlation coefficient, $r$, represents the strength of the correlation between intensity of response for the behavior and setting videos. Total Positive Affect does not include alert or attentive. ${ }^{*} \mathrm{p}<0.05,{ }^{*} \mathrm{p}<0.01$.

\begin{tabular}{|c|c|c|c|c|c|}
\hline & Feeding & Curious & Aggressive & \multirow[t]{2}{*}{$F(2,507)$} & \multirow[t]{2}{*}{$\mathrm{r}$} \\
\hline \multicolumn{4}{|c|}{ Estimated Marginal Mean (SE) } & & \\
\hline Positive Affect & & & & & \\
\hline Alert & $3.90(0.1)^{\mathrm{a}}$ & $4.30(0.1)^{\mathrm{b}}$ & $4.38(0.1)^{\mathrm{b}}$ & $11.56^{* *}$ & $0.47^{* *}$ \\
\hline Attentive & $3.92(0.1)^{\mathrm{a}}$ & $4.19(0.1)^{\mathrm{b}}$ & $4.36(0.1)^{\mathrm{b}}$ & $8.84^{* *}$ & $0.51^{* *}$ \\
\hline Enthusiastic & $3.13(0.1)^{\mathrm{b}}$ & $2.74(0.1)^{\mathrm{a}}$ & $2.53(0.1)^{\mathrm{a}}$ & $12.61^{* *}$ & $0.47^{* *}$ \\
\hline Excited & $3.36(0.1)^{\mathrm{b}}$ & $3.07(0.1)$ & $3.04(0.1)^{\mathrm{a}}$ & $3.81^{*}$ & $0.46^{* *}$ \\
\hline Interested & $3.71(0.1)^{\mathrm{b}}$ & $3.44(0.1)^{\mathrm{b}}$ & $2.86(0.1)^{\mathrm{a}}$ & $23.40^{* *}$ & $0.42^{* *}$ \\
\hline Total PA & $10.20(0.2)^{\mathrm{c}}$ & $9.23(0.2)^{\mathrm{b}}$ & $8.45(0.2)^{\mathrm{a}}$ & $16.83^{* *}$ & $0.55^{* *}$ \\
\hline \multicolumn{6}{|l|}{ Negative Affect } \\
\hline Distressed & $2.22(0.1)^{\mathrm{a}}$ & $3.09(0.1)^{\mathrm{b}}$ & $3.67(0.1)^{\mathrm{c}}$ & $71.72^{* *}$ & $0.42^{* *}$ \\
\hline Hostile & $1.81(0.1)^{\mathrm{a}}$ & $2.13(0.1)^{\mathrm{b}}$ & $2.62(0.1)^{\mathrm{c}}$ & $26.48^{* *}$ & $0.60^{* *}$ \\
\hline Jittery & $2.38(0.1)^{\mathrm{a}}$ & $3.27(0.1)^{b}$ & $3.66(0.1)^{\mathrm{c}}$ & $55.58^{* *}$ & $0.36^{* *}$ \\
\hline Nervous & $2.72(0.1)^{\mathrm{a}}$ & $3.51(0.1)^{\mathrm{b}}$ & $3.86(0.1)^{\mathrm{c}}$ & $41.41^{* *}$ & $0.36^{* *}$ \\
\hline Scared & $2.71(0.1)^{\mathrm{a}}$ & $3.48(0.1)^{\mathrm{b}}$ & $3.91(0.1)^{\mathrm{c}}$ & $43.50^{* *}$ & $0.40^{* *}$ \\
\hline Total NA & $11.85(0.3)^{\mathrm{a}}$ & $15.47(0.4)^{\mathrm{b}}$ & $17.71(0.3)^{\mathrm{c}}$ & $75.45^{* *}$ & $0.48^{* *}$ \\
\hline
\end{tabular}

Table 3. Univariate general linear model for the impact of previous bear safety education on an individual's in-the-moment decision for both video treatments. Note: ${ }^{*} \mathrm{p}<0.05 * * \mathrm{p}<0.01$.

\begin{tabular}{rcccc}
\hline Action & df & $\mathrm{F}$ & $\mathrm{B}(\mathrm{SE})$ & Adjusted R $^{2}$ \\
\hline Corrected model & 19,1021 & $12.8^{* *}$ & & 0.18 \\
Intercept & 1,1021 & $46.5^{* *}$ & $3.47(0.6)$ & \\
Video treatment & 11,1021 & 1.0 & & \\
Taught bear safety-No & 2,1021 & $8.0^{* *}$ & $-1.17(0.4)$ & \\
Feelings toward bears & 1,1021 & $23.8^{* *}$ & $0.23(0.0)$ & \\
Age & 1,1021 & $19.3^{* *}$ & $0.02(0.0)$ & \\
Alert & 1,1021 & $13.9^{* *}$ & $0.33(0.1)$ & \\
Attentive & 1,1021 & $10.7^{* *}$ & $0.31(0.1)$ & \\
Interested & 1,1021 & $12.2^{* *}$ & $0.22(0.1)$ & \\
Hostile & 1,1021 & $9.7^{* *}$ & $-0.19(0.1)$ &
\end{tabular}

\section{Current emotion}

For the impact of emotion on a respondent's in-the-moment decision, four independent variables were significant, $F(15,1002)$ $=6.34, p<0.001$, adjusted $\mathrm{R}^{2}=0.086$. These were the affective items of attentive, interested, hostile, and scared. The adjusted $\mathrm{R}^{2}$ value of 0.07 suggests the model explains approximately $7 \%$ of the variation in the anticipated impact of current emotion on an in-the-moment decision and as a result, does little to predict this impact. However, for all four significant independent variables, an increase in the intensity of the affective response resulted in an increased impact of emotion on decision making $(F[1,1002]$ $=$ Attentive: $8.99, \mathrm{~B}=0.02$; Interested: $7.25, \mathrm{~B}=0.18$; Hostile: $16.35, \mathrm{~B}=0.29$ Scared: $13.34, \mathrm{~B}=0.26$ )

\section{Previous experience}

Last, for the impact of previous experience on a respondent's inthe-moment decision, six independent variables were significant, $F(17,1002)=8.00, p<0.001$, adjusted $\mathrm{R}^{2}=0.104$. These were having seen a bear, general feelings toward bears, self-reported grade in bear safety, and the affective items of attentive, enthusiastic, and scared. Respondents who had not previously seen a bear of any species reported a lower impact of previous experience, $F(1,1002)=12.11, \mathrm{~B}=-0.66)$. More positive feelings toward bears $(B=0.22)$, higher bear safety grades $(B=0.09)$, and increasing levels of attentiveness $(\mathrm{B}=0.284)$, enthusiasm $(\mathrm{B}=$ $0.22)$, and fear $(B=0.35)$ all resulted in an increased impact of previous experience on decision making.

\section{Strategies to encourage safe behavior}

Additionally, we asked respondents to rank the importance of three strategies to encourage safe behavior. Using the approaches to overcoming affective shortcuts detailed by Wilson (2008), we included (1) being told why instructions or policies are what they are, (2) relating with the goals of park managers, and (3) using your own personal values to justify safe behavior. Across responses, $66.3 \%$ selected strategy 1 as the most important, $21.3 \%$ selected strategy 2 as the most important, and $12.4 \%$ selected strategy 3 as the most important.

\section{DISCUSSION}

We used a mixed methods (primarily quantitative), exploratory sequential design, to understand both the immediate emotional reactions upon viewing bears in different scenarios and the role of emotional reactions in individuals' ability to behave appropriately. Survey results suggest respondents are generally aware of appropriate behavior around brown bears, but affective responses may hamper their ability to behave accordingly.

\section{Encouraging appropriate behavior}

With increasing interest in outdoor recreation, rising visitation levels to parks and protected areas, and expanding brown bear populations, the frequency of human-bear interactions may continue to increase. Unfortunately, many individuals may not know how to behave and could cause injury to themselves or a bear.

As suggested by the linear models in this study, different encounter scenarios may result in different behavioral strategies. Scenarios 
that result in feelings of attentiveness, hostility, and fear may decrease the impact of safety education on behavioral choices while increasing the impact of previous experiences and current emotion. However, among setting treatments, a subadult in a meadow produced the highest level of attentiveness and levels of hostility and fear were both highest in response to a sow and cubs in a meadow. Although these affective responses are highly appropriate for the given scenario, they unfortunately increase the impact of current emotion on decision making.

Similarly, among behavior treatments, the level of attentiveness was higher for curious and aggressive behaviors than feeding behavior and levels of hostility and fear both increased as the behavior treatment increased in aggressiveness. So, as the scenarios become more dangerous and require careful decisions, the more impact emotion has on behavior. In order to continue improving the current effectiveness of bear safety and education efforts, the impact of affective responses must be addressed. Respondents' ranking of potential education strategies stress the potential benefits of explaining to park visitors why instructions and policies were put in place and how they work.

\section{Affective responses}

Encouragingly, respondents viewing a sow and cubs in a meadow reported the highest levels of negative affect, followed by a subadult in a stream. These two treatments represent the setting with the highest potential for danger and the setting with the most erratic behavior (Herrero 1976, 2018). Interestingly, those viewing a sow and cubs from a platform reported the lowest levels of negative affect. This suggests that despite high levels of positive affect, respondents recognized the potential for danger when viewing a sow and cubs while exposed in a meadow. When viewing from a platform, a significant portion of this concern was presumably alleviated, and respondents reported a largely positive experience.

Further, while total levels of negative affect were slightly higher in a stream or meadow than from a platform, total levels of positive affect were slightly lower in a stream than a meadow or from a platform. This lower level of positive affect may have been a result of the focal bears being partially obscured by water, the orientation or movement of bears displayed in the videos, or unfamiliarity among respondents with bears in a stream setting.

As expected, respondents who viewed increasingly aggressive behaviors, reported lower levels of positive affect and higher levels of negative affect. This trend suggests respondents were largely able to correctly identify the behaviors presented. The fact that alertness and attentiveness were higher following aggressive behavior than curious or feeding behavior highlights a limitation of the PANAS scale. Although researchers traditionally list these items among positive affect, they may have been interpreted among respondents as more neutral, or even negative. For example, being alert and attentive is an expected human response to aggressive behaviors from bears and could be perceived negatively. As a result, analysis of the PANAS scale has been primarily conducted at the item level, rather than the factor level.

\section{Behavioral responses}

Objective appropriateness

Objective appropriateness for the 15 listed actions was determined using recommendations outlined by Herrero (2018) and
Masterson (2016) and incorporated into Table 4 and the discussion in this section. Those listed as "Inappropriate" are not recommended in any scenario because they could result in personal injury, could promote bear habituation, or would simply not be effective. Those listed as "Appropriate" are recommended across all scenarios. The appropriateness of those listed as "Depends" ${ }^{[4]}$ can depend on several factors, but mainly the bear's behavior and the species of bear.

Table 4. Perceived and objective appropriateness of listed actions. Note: Perceived appropriateness is based on average ratings across all setting treatments. Ratings were on a 7-point Likert scale $(1=$ extremely inappropriate, $4=$ neither appropriate nor inappropriate, 7 = extremely appropriate). Objective appropriateness was determined based on recommendations outlined by Herrero (2018) and Masterson (2016).

\begin{tabular}{rcc}
\hline Listed Action & $\begin{array}{c}\text { Perceived } \\
\text { Appropriateness } \\
\text { Mean (SD) }\end{array}$ & $\begin{array}{c}\text { Objective } \\
\text { Appropriateness }\end{array}$ \\
\hline Approach the bear & $1.75(1.6)$ & Inappropriate \\
Back away slowly & $5.57(1.6)$ & Appropriate \\
Climb a tree & $2.99(1.9)$ & Inappropriate \\
Kick or punch the bear & $2.36(1.9)$ & Depends \\
Group together & $4.33(2.0)$ & Appropriate \\
Play dead & $3.89(2.0)$ & Depends \\
Run away & $2.87(2.1)$ & Inappropriate \\
Depends \\
Shoot the bear & $3.31(2.2)$ & Inappropriate \\
Shout or make noise & $2.52(2.1)$ & Depends \\
Spray with bear spray & $2.86(2.1)$ & Depends \\
Stand still and wait & $4.77(1.8)$ & Inappropriate \\
Throw food into the woods & $3.27(2.0)$ & Depends \\
Throw things at the bear & $2.18(1.8)$ & Inappropriate \\
Try to hide & $4.09(2.0)$ & Depends \\
\hline Walk around the bear & $4.64(2.1)$ &
\end{tabular}

Likelihood and perceived appropriateness

The most directly applicable results of this study came from the difference between likelihood and perceived appropriateness of potential actions. In cases where likelihood exceeded appropriateness, individuals may have known the intended behavior could have resulted in injury, but were potentially unable to overcome their emotional and affective responses to the bear encounter. Such scenarios included running away from a boar in a meadow, throwing things at a subadult in a meadow, and throwing food into the woods to lure a sow and cubs away from a platform. These three behaviors then, are areas of concern within bear safety and management. Public perception regarding these behaviors may prove difficult to overcome. In order to do so, efforts must be made to encourage further internalization of not only the danger associated with these behaviors, but how to behave appropriately in such scenarios as well. All park visitors and area residents must fully understand the benefits of appropriate actions and the consequences of inappropriate actions.

When likelihood exceeded appropriateness, individuals were likely aware that their actions may not have been appropriate. However, when the opposite was true, individuals believed the behavior to be appropriate but were unable to act accordingly. In such cases, individuals may understand the importance of 
behaving in such a manner, but current emotional and affective state encourage behaving otherwise. Some of these behaviors were shooting a subadult in a meadow, backing away slowly or playing dead to avoid a sow and cubs in a stream, and backing away slowly when viewing a boar from a platform. Further, all seven significant differences among behavior treatments fall into this category as well. These include playing dead to avoid a feeding bear, climbing a tree, playing dead, waiting for the bear to leave, or walking around the bear to avoid a curious bear, and climbing a tree or walking around the bear to avoid an aggressive bear.

Similar to inappropriate behaviors in the former category, behaviors in this latter category that were appropriate, such as backing away slowly or walking around a sow and cubs in a stream, require management and safety instruction to emphasize the benefits to such behavior and the reasoning behind its effectiveness. Experiential training through photographs, videos, or virtual reality could help park visitors and area residents to imagine such scenarios and practice behaving appropriately, weakening these affective shortcuts. If needed, examples of worstcase scenarios may provide a significant reminder of potential consequences. Most likely because of the novelty of the experience, viewing a sow and cubs in a stream resulted in the most behaviors in this category and as a result, requires additional attention.

An additional area of concern is the fact that several actions in this latter category could be inappropriate or dangerous (Herrero 2018). Climbing a tree, responding aggressively (i.e., shooting the bear or throwing things at the bear), throwing food to lure the bear away, and running away are all seen among the general American public as appropriate but are rarely so, and could result in injury or death to either party. According to survey results, these actions were more appropriate than likely, making them not yet problematic, as respondents were generally less likely to behave in such a way. In order to prevent these actions from becoming a problem, managers and staff must continue to reinforce the inappropriateness of these actions, explaining the specific factors leading to their inefficacy.

\section{Management implications}

From these results and suggestions provided by respondents, we developed eight recommendations to improve the overall effectiveness of safety training and education efforts regarding brown bears. These recommendations encourage improvements in the availability of information, hands-on training experiences, and explicit reasoning behind policies and regulations. By incorporating aspects of behavioral psychology, we believe these recommendations will be valuable and effective in encouraging appropriate behavior.

1. All bear safety education should warn of potential affective responses such as fear, hostility, and attentiveness that may occur during an encounter and remind users to be aware of their emotions, and to ultimately remain calm.

2. Education efforts should continue to address the appropriateness of uncertain or popular behaviors, including climbing a tree, running away, trying to hide, grouping together, and walking around the bear.

3. Regions with established brown bear populations, as well as those on the edge of dispersing populations, should release bear safety public service announcements across several forms of media to reach the widest audience possible.

4. Place educational materials, e.g., signs, pamphlets, interactive displays, etc., at various locations outside of park visitor's centers at other access areas.

5. Use photographs, videos, and virtual reality experiences to incorporate various scenarios and bear behaviors in preparing users for potential bear encounters

6. Provide logic and reasoning behind policies, regulations, and behavioral instructions, including potential negative results.

7. Pay special attention to overly confident individuals, those with more experience, and those more likely to respond aggressively, particularly younger males.

8. If necessary and feasible, mandatory bear safety training prior to park admission may be an effective solution. If not, consider implementing a mandatory bear safety lesson or video prior to purchasing park passes online.

\section{Limitations}

Threats to internal validity

Although the PANAS scale has been well tested and items were chosen to best reflect the viewing experience, its use and abbreviation only approximates the range of potential affective responses. A representative sample was collected with adequate sample size, but Qualtrics users and those willing to take the survey may not be entirely representative of the general American public, or those visiting national parks or other protected areas where bear viewing may occur. Although statistically accounted for by appropriate analysis, the repeated use of scales within the survey may have resulted in a testing effect, or dependency between measures not entirely accounted for by the statistical procedures.

\section{Threats to external validity}

For feasibility, survey respondents were provided with videos representing each scenario as opposed to firsthand experience. As a result, affective responses may have been limited or dulled. The online survey format also allowed respondents to pause and reflect prior to answering and potentially select answers believed to be correct rather than true. Last, despite significant effort to minimize confounding variables among videos, some were unavoidable and may have unintentionally altered affective responses.

\section{Future research}

Research on emotional reactions to viewing wildlife, especially carnivores, has been limited in scope. Many previous studies have addressed feelings toward carnivores (e.g., Farber and Hall 2007, Raadik and Cottrell 2007, Jacobs et al. 2012a, b) or opinions regarding potential management action (e.g., McFarlane et al. 2007, Glikman et al. 2012, Johansson et al. 2012, Hayman et al. 2014, Fefer et al. 2020), but the focus on how these reactions impact behavior is currently understudied. This study begins to address the current knowledge gap, but more importantly, provides a foundation for continued exploration. Future research could use participant observations and onsite qualitative interviews to further understand complex emotional responses 
beyond what quantitative questionnaires allow. It is also important to test the applicability of virtual reality technology in representing onsite encounters. Although still more contrived than firsthand experience, this developing technology could allow park managers and bear safety administrators to accurately represent different scenarios and encourage appropriate behavior despite affective responses. Further research in virtual reality could also be used to advance visual methods within park and visitor management research.

An additional area of interest is the applicability of this research to other settings or wildlife species. Videos and questions included in this survey were targeted at brown bears, but behavioral similarities and misidentification suggest results may be highly transferable to black bears. Future research efforts should assess this degree of transferability to not only black bears, but entirely different contexts as well, such as African safaris, tiger viewing in India, underwater at coral reefs, or even deer hunting. Although it would need to be tested, we anticipate similar results across contexts.

\section{CONCLUSION}

Not only do affective responses differ across various bear encounter scenarios, these affective responses can potentially discourage appropriate behavior while encouraging inappropriate behavior. Scenarios that produce intense affective responses are often the most dangerous, but significantly increase the impact of emotion on decision making. Managers of relevant parks and protected areas must continue to educate visitors on safe behavior around bears while further detailing the reasoning and logic behind policies and encouraged behaviors. Although rarely feasible, the ideal solution would be to conduct mandatory training sessions at all relevant parks and protected areas prior to admission, including further emphasis on appropriate behavior. Survey respondents also suggested public service announcements, further online training or information regarding safe behavior, video and virtual reality practice encounters, education on understanding bear behavior, and cautionary tales, as potential methods to encourage appropriate behavior; all of which could provide feasible and effective improvements to bear safety education.

${ }^{[1]}$ The full list of questions asked during informal interviews is available by contacting the corresponding author.

${ }^{[2]}$ The full survey is available online at https://doi.org/10.6084/m9. figshare.c.5296201.v1

${ }^{[3]}$ Please contact the corresponding author for the full table of results and associated post hoc tests.

${ }^{[4]}$ Please contact the corresponding author for a complete list of the criteria and justification of these categories.

Responses to this article can be read online at: https://www.ecologyandsociety.org/issues/responses. php/12410

\section{Acknowledgments:}

We would like to thank YouTube users GroundTruthTrekking and Photoguide for the videos used in the behavior treatments.

\section{Data Availability:}

The datalcode that support the findings of this study are available on request from the corresponding author, JN. The datalcode are not publicly available because they contain information that could compromise the privacy of research participants. In accordance with agency and institutional guidelines, the U.S. National Park Service and the Office of Management and Budget approved the field protocols (OMB\# 1024-0224). The Clemson University Office of Research Compliance reviewed the protocols and determined that the research activities involving human participants qualified as Exempt (IRB\#2018000010) under category 2 in accordance with federal regulations 45 CFR 46.104(d).

\section{LITERATURE CITED}

Aumiller, L. D., and C. A. Matt. 1994. Management of McNeil River State Game Sanctuary for viewing of brown bears. Bears: Their Biology and Management 9:51-61. https://doi.org/10.2307/3872684

Bateson, J. E. G., and M. K. Hui. 1992. The ecological validity of photographic slides and videotapes in simulating the service setting. Journal of Consumer Research 19(2):271-281. https://doi. org/10.1086/209301

Byrne, B. M. 2008. Testing for multiple equivalence of a measuring instrument: a walk through the process. Psicothema 20(4):872-882.

Cabrera, N. L. 2011. Using a sequential exploratory mixedmethod design to examine racial hyperprivilege in higher education. New Directions for Institutional Research 2011 (151):77-91. https://doi.org/10.1002/ir.400

Crawford, J. R., and J. D. Henry. 2004. The positive and negative affect schedule (PANAS): construct validity, measurement properties and normative data in a large non-clinical sample. British Journal of Clinical Psychology 43(3):245-265. https://doi. org/10.1348/0144665031752934

Cribbs, T., R. Sharp, and M. Brownlee. 2019. Evaluating the influence of photo order on park visitors' perceptions of crowding at Buffalo National River. Leisure Sciences. https://doi. org/10.1080/01490400.2019.1655685

Crocker, P. R. E. 1997. A confirmatory factor analysis of the Positive Affect Negative Affect Schedule (PANAS) with a youth sport sample. Journal of Sport and Exercise Psychology 19 (1):91-97. https://doi.org/10.1123/jsep.19.1.91

Dillman, D. A. 2011. Mail and internet surveys: the tailored design method: 2007 update with new internet, visual, and mixed-mode guide. Second edition. John Wiley and Sons, Hoboken, New Jersey, USA.

Ekkekakis, P. 2012. Affect, mood, and emotion. Pages 321-332 in G. Tenenbaum, R. C. Eklund, and A. Kamata, editors. Measurement in sport and exercise psychology. Human Kinetics, 
Champaign, Illinois, USA. https://doi.org/10.5040/9781492596332. ch-028

Farber, M. E., and T. E. Hall. 2007. Emotion and environment: visitors' extraordinary experiences along the Dalton Highway in Alaska. Journal of Leisure Research 39(2):248-270. https://doi. org/10.1080/00222216.2007.11950107

Fefer, J., J. Hallo, R. Dvorak, M. Brownlee, R. Collins, and E. Baldwin. 2020. Pictures of polar bears: using visitor employed photography to identify experience indicators in the Arctic National Wildlife Refuge. Journal of Environmental Management 269(1):110779. https://doi.org/10.1016/j.jenvman.2020.110779

Freimund, W. A., J. J. Vaske, M. P. Donnelly, and T. A. Miller. 2002. Using video surveys to access dispersed backcountry visitors' norms. Leisure Sciences 24(3-4):349-362. https://doi. org/10.1080/01490400290050790

Glikman, J. A., J. J. Vaske, A. J. Bath, P. Ciucci, and L. Boitani. 2012. Residents' support for wolf and bear conservation: the moderating influence of knowledge. European Journal of Wildlife Research 58(1):295-302. https://doi.org/10.1007/s10344-011-0579$\underline{x}$

Gunther, K. A., and H. E. Hoekstra. 1998. Bear-inflicted human injuries in Yellowstone National Park, 1970-1994. Ursus 10:377-384.

Halcomb, E. J., and P. M. Davidson. 2006. Is verbatim transcription of interview data always necessary? Applied Nursing Research 19(1):38-42. https://doi.org/10.1016/j.apnr.2005.06.001

Hammitt, W. E., D. N. Cole, and C. A. Monz, 2015. Wildland recreation: ecology and management. John Wiley \& Sons, Hoboken, New Jersey, USA.

Haroldson, M. A., and K. A. Gunther. 2013. Roadside bear viewing opportunities in Yellowstone National Park: characteristics, trends, and influence of whitebark pine. Ursus 24 (1):27-41. https://doi.org/10.2192/URSUS-D-10-00036.1

Hayman, R. B., R. G. Harvey, F. J. Mazzotti, G. D. Israel, and A. R. Woodward. 2014. Who complains about alligators? Cognitive and situational factors influence behavior toward wildlife. Human Dimensions of Wildlife 19(6):481-497. https://doi. org/10.1080/10871209.2014.918218

Herrero, S. 1976. Conflicts between man and grizzly bears in the national parks of North America. Bears: Their Biology and Management 3:121-145. https://doi.org/10.2307/3872761

Herrero, S. 2018. Bear attacks: their causes and avoidance. Third edition. Lyons Press. Guilford, Connecticut, USA.

Herrero, S., T. Smith, T. D. DeBruyn, , K. Gunther, and C. A. Matt. 2005. From the field: brown bear habituation to peoplesafety, risks, and benefits. Wildlife Society Bulletin 33(1):362-373. https://doi.org/10.2193/0091-7648(2005)33[362:ftfbbh]2.0.co:2

Jacobs, M. H., P. Fehres, and M. Campbell. 2012a. Measuring emotions toward wildlife: a review of generic methods and instruments. Human Dimensions of Wildlife 17(4):233-247. https://doi.org/10.1080/10871209.2012.680175

Jacobs, M. H., J. J. Vaske, and J. M. Roemer. 2012b. Toward a mental systems approach to human relationships with wildlife: the role of emotional dispositions. Human Dimensions of Wildlife 17(1): 4-15. https://doi.org/10.1080/10871209.2012.645123

Jochum, K. A., A. A. Kliskey, K. J. Hundertmark, and L. Alessa. 2014. Integrating complexity in the management of humanwildlife encounters. Global Environmental Change 26(1):73-86. https://doi.org/10.1016/j.gloenvcha.2014.03.011

Johansson, M., M. Sjöström, J. Karlsson, and R. Brännlund. 2012. Is human fear affecting public willingness to pay for the management and conservation of large carnivores? Society \& Natural Resources 25(6):610-620. https://doi.org/10.1080/08941920.2011.622734

Kahneman, D., and A. Tversky. 1973. On the psychology of prediction. Psychological Review 80(4):237-251. https://doi. org/10.1037/h0034747

Kline, R. B. 2012. Assumptions in structural equation modeling. Pages 111-125 in R. H. Hoyle, editor. Handbook of structural equation modeling. Guilford Press, New York, New York, USA.

Laven, D. N., and D. H. Krymkowski. 2005. The relationship between visitor-based standards of quality and existing conditions in parks and outdoor recreation. Leisure Sciences 27 (2):157-173. https://doi.org/10.1080/01490400590912060

Manning, R. E., and W. A. Freimund. 2004. Use of visual research methods to measure standards of quality for parks and outdoor recreation. Journal of Leisure Research 36(4):557-579. https://doi. org/10.1080/00222216.2004.11950036

Masterson, L. 2016. Living with bears handbook. Second edition. PixyJack, Masonville, Colorado, USA.

McFarlane, B. L., R. Stumpf-Allen, and D. O. T. Watson. 2007. Public acceptance of access restrictions to grizzly bear (Ursus arctos) country. Human Dimensions of Wildlife 12(4):275-287. https://doi.org/10.1080/10871200701195555

Monz, C., and Y. F. Leung. 2006. Meaningful measures: developing indicators of visitor impact in the national park service inventory and monitoring program. George Wright Forum 23(2):17-27.

Nettles, J., M. Brownlee, R. Sharp, S. Jackson, and D. Dagan 2020. Evaluation of the bear viewing experience and associated thresholds at Katmai National Park \& Preserve and Lake Clark National Park \& Preserve. Technical report submitted to the U.S. National Park Service. In fulfillment of Cooperative Agreement No. P17AC01038.

Newman, P., J. L. Marion, and K. Cahill. 2001. Integrating resource, social, and managerial indicators of quality into carrying capacity decision-making. George Wright Forum 18 (3):28-40

Penteriani, V., J. V. López-Bao, C. Bettega, F. Dalerum, M. Mar Delgado, K. Jerina, I. Kojola, M. Krofel, and A. Ordiz. 2017. Consequences of brown bear viewing tourism: a review. Biological Conservation 206:169-180. https://doi.org/10.1016/j.biocon.2016.12.035

Quartz, S. R. 2009. Reason, emotion and decision-making: risk and reward computation with feeling. Trend in Cognitive Sciences 13(5):209-215. https://doi.org/10.1016/j.tics.2009.02.003 
Raadik, J., and S. Cottrell. 2007. Wildlife value orientations: an Estonian case study. Human Dimensions of Wildlife 12 (5):347-357. https://doi.org/10.1080/10871200701555378

Schmukle, S. C., B. Egloff, and L. R. Burns. 2002. The relationship between positive and negative affect in the positive and negative affect schedule. Journal of Research in Personality 36:463-475. https://doi.org/10.1016/S0092-6566(02)00007-7

Seidman, I. 2012. Interviewing as qualitative research: a guide for researchers in education and the social sciences. Teachers College Press, New York, New York, USA.

Slovic, P., E. Peters, M. L. Finucane, and D. G. MacGregor. 2005. Affect, risk, and decision making. Health Psychology 24(4S):S35S40. https://doi.org/10.1037/0278-6133.24.4.S35

Thompson, E. R. 2007. Development and validation of an internationally reliable short-form of the Positive and Negative Affect Schedule (PANAS). Journal of Cross-Cultural Psychology 38(2):227-242. https://doi.org/10.1177/0022022106297301

Tversky, A., and D. Kahneman. 1973. Availability: a heuristic for judging frequency and probability. Cognitive Psychology 5 (2):207-232. https://doi.org/10.1016/0010-0285(73)90033-9

U.S. Census Bureau (USCB). 2020. ACS demographic and housing estimates. USCB, Washington, D.C., USA. [online] URL: https:// data.census. gov/cedsci/table? $\mathrm{q}=$ demographics $\&$ hidePreview=false $\&$ tid $=$ $\underline{\text { ACSDP1Y2018.DP05\&vintage }=2018}$

Vaske, J. J. 2008. Survey research and analysis: applications in parks, recreation, and human dimensions. Venture, State College, Pennsylvania, USA.

Verbos, R. I., C. A. B. Zajchowski, M. T. J. Brownlee, and J. C. Skibins. 2018. 'I'd like to be just a bit closer': wildlife viewing proximity preferences at Denali National Park \& Preserve. Journal of Ecotourism 17(4):409-424. https://doi.org/10.1080/14724049.2017.1410551

Watson, D., L. A. Clark, and A. Tellegen. 1988. Development and validation of brief measures of positive and negative affect. Journal of Personality and Social Psychology 54(6):1063-1070. https://doi.org/10.1037/0022-3514.54.6.1063

Wilson, R. S. 2008. Balancing emotion and cognition: a case for decision aiding in conservation efforts. Conservation Biology 22 (6):1452-1460. https://doi.org/10.1111/j.1523-1739.2008.01016.x

Zajchowski, C., M. Brownlee, and N. Furman. 2016. The dialectical utility of heuristic processing in outdoor adventure education. Journal of Outdoor Recreation, Education, and Leadership 8(2):118-134. https://doi.org/10.18666/JOREL-2016$\underline{\mathrm{V} 8-\mathrm{I} 2-7697}$ 